Supporting Information

\title{
Tissue-mimetic dielectric actuators: Free-standing, stable, and solvent-free
}

\author{
Vahid Karimkhani ${ }^{1}$, Mohammad Vatankhah-Varnosfaderani ${ }^{1 *}$, Andrew N. Keith ${ }^{1}$, Erfan \\ Dashtimoghadam ${ }^{1}$, Benjamin J. Morgan ${ }^{1}$, Michael Jacobs ${ }^{2}$, Andrey V. Dobrynin ${ }^{2}$, Sergei S. \\ Sheiko ${ }^{1 *}$ \\ ${ }^{1)}$ Department of Chemistry, University of North Carolina, Chapel Hill, North Carolina, 27599- \\ 3290, United States \\ ${ }^{2)}$ Department of Polymer Science, University of Akron, Akron, Ohio, 44325, United States
}

Corresponding authors: $\quad$ sergei@email.unc.edu

$\underline{\text { mvatan@live.unc.edu }}$ 


\section{S1 Synthesis and Characterization}

S1.1 Materials Monomethacryloxypropyl-terminated poly(dimethylsiloxane) (MCR-M11, average molar mass $1000 \mathrm{~g} / \mathrm{mol}$, degree polymerization $(\mathrm{DP})=14$, dispersity, $Đ=1.15$ ) was obtained from Gelest. Methyl methacrylate (MMA, 99\%) was obtained from Acros. Both monomers purified by passing through basic alumina columns to remove inhibitor. Copper(I) chloride $(\mathrm{CuCl}, \geq 99.995 \%)$, tris[2-(dimethylamino)ethyl]amine (Me6TREN), ethylene glycol (anhydrous, 99.8\%), Triethylamine ( $\geq 99.5 \%$ ), $\alpha$-Bromoisobutyryl bromide (98\%), tetrahydrofuran (THF), p-xylene (PX) and toluene were purchased from Sigma Aldrich and used as received.

S1.2 Synthesis of ethylene bis(2-bromoisobutyrate) (2-BiB). The synthesis was adapted from a literature with some modifications. Ethylene glycol (14.43 g, $0.23 \mathrm{~mol})$ was added under nitrogen to a flask containing a magnetic stirring bar and placed in an ice bath. Anhydrous THF was added into the flask. Triethylamine $(60 \mathrm{~mL}, 3$ eq.) was added to the reaction mixture. 2- Bromoisobutyryl bromide (120 g, 2.2 eq.) was added dropwise to the reaction mixture with a syringe pump. After a complete addition, the reaction mixture stirred at $0{ }^{\circ} \mathrm{C}$ for extra one hour and then at ambient temperature overnight. The mixture was filtered and the volatile compounds were removed by rotary evaporation. The resulting brown solution was dissolved in chloroform and treated with $1 \mathrm{M}$ $\mathrm{HCl}$ solution, saturated $\mathrm{NaHCO}_{3}$ solution and three times with deionized water. The organic layer was dried over dry $\mathrm{MgSO}_{4}$. The volatile compounds were removed with vacuum to give a light brown solid. This sample passed through a column to purify and achieve pure creamy white crystal after drying sample. 


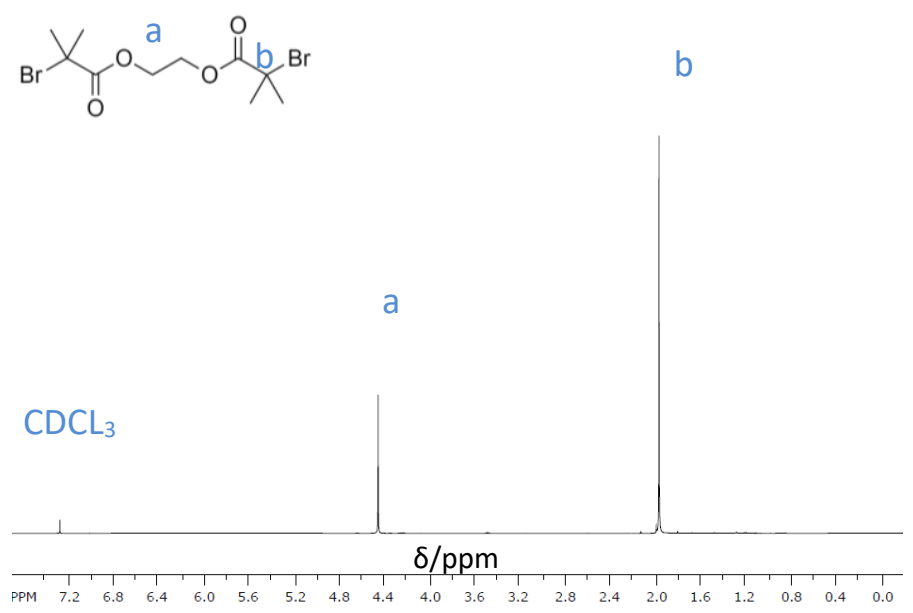

Fig. S1. ${ }^{1} \mathrm{H}-\mathrm{NMR}$ spectrum of purified 2-BiB in $\mathrm{CDCl}_{3}(400$ $\mathrm{MHz}$.

S1.3 Synthesis of poly(dimethylsiloxane) bottlebrushes. ${ }^{1}$ A $100 \mathrm{~mL}$ Schlenk flask equipped with a stir bar was charged with 2-BiB (14.4 mg, $40.0 \mu \mathrm{mol})$, MCR-M11 (45.0 g, $45.0 \mathrm{mmol})$, Me6TREN (18.4 mg, $22.2 \mu \mathrm{L}, 80.0 \mu \mathrm{mol})$, and p-xylene $(45.0 \mathrm{~mL})$. The solution was bubbled with dry nitrogen for $1.5 \mathrm{hr}$, then $\mathrm{Cu}(\mathrm{I}) \mathrm{Cl}(7.9 \mathrm{mg}, 79.8 \mu \mathrm{mol})$ was quickly added to the reaction mixture under nitrogen atmosphere. The flask was sealed, back-filled with nitrogen, purged for 15 minutes, and then immersed in a $45^{\circ} \mathrm{C}$ oil bath. The polymerization was stopped after $\sim 12$ hours at $\sim 80 \%$ monomer conversion, resulting in a bottlebrush PDMS polymer with DP of the backbone $\left(n_{b b}\right)$ $\sim 900$. The polymer was precipitated three-five times with DMF from DCM solution to purify residual macromonomer and passed through neutral aluminum oxide columns to remove residual catalyst. The resulting purified polymer was dried under vacuum at room temperature until a constant mass was reached. 


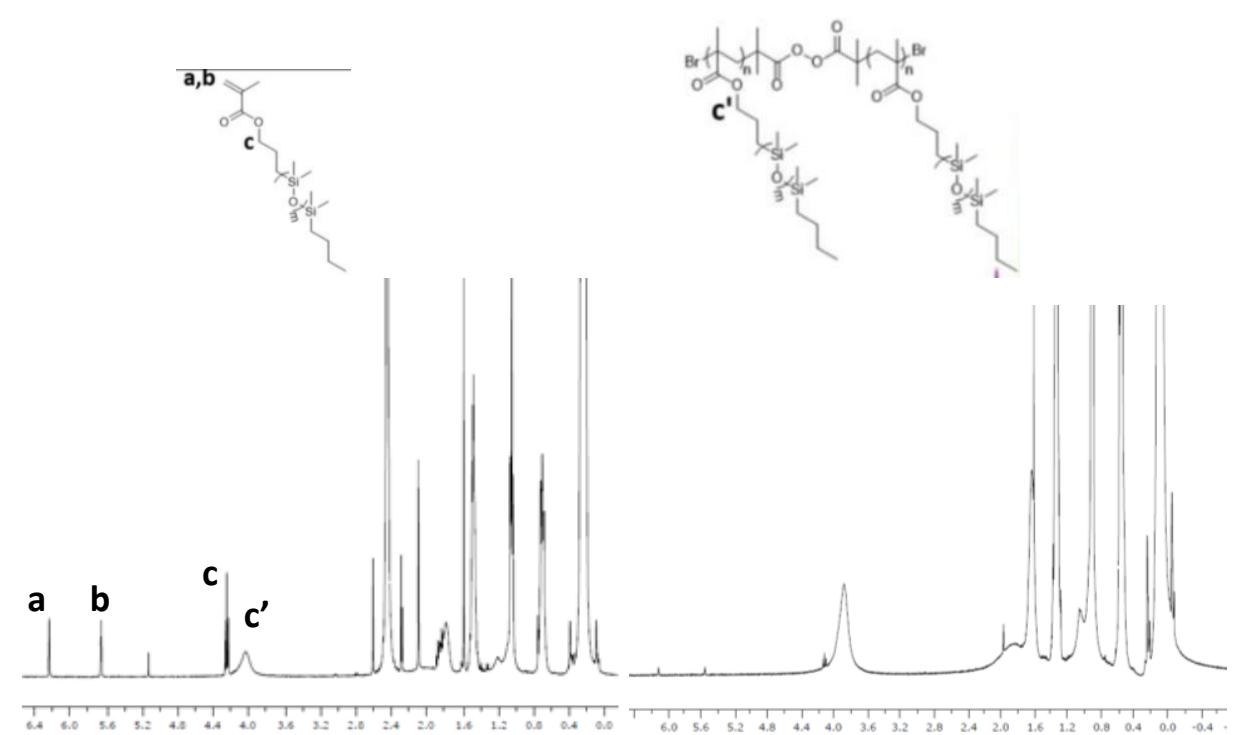

Fig. S2. (a) ${ }^{1} \mathrm{H}-\mathrm{NMR}$ of $\mathrm{S} 1.2$ reaction mixture at $79 \%$ conversion (400 MHz, CDCl3): 6.22 , $5.66(\mathrm{CH} 2=\mathrm{C}(\mathrm{CH} 3) \mathrm{C}=\mathrm{O}$, PDMS macromonomer, s, 2H), $4.22(\mathrm{CO}-\mathrm{OCH} 2$, PDMS macromonomer, t, $2 \mathrm{H}), 3.96(\mathrm{CO}-\mathrm{OCH} 2$, reacted PDMS bottlebrush. $\mathrm{m}, 2 \mathrm{H})$, conversion = $\left[\operatorname{area}\left(c+c^{\prime}\right)-\operatorname{area}(2 b)\right] / \operatorname{area}\left(c+c c^{\prime}\right)=79 \%$. (b) The NMR spectra of purified samples after washing through percipitation of polymer in the DMF, from dichloromethane solution.

S1.4 A-bbB-A Linear-Bottlebrush-Linear plastomer synthesis. ${ }^{1}$ To prepare A-bbB-A block copolymers, synthesized PDMS bottlebrushes were used as ATRP initiators to grow linear PMMA side-blocks at both ends. With a similar procedure to Section S1.3, marco-initiator, monomer and Me6TREN were dissolved in toluene, degassed and followed with the addition of $\mathrm{CuCl}$. The compositions of A-bbB-A copolymers in the reaction flasks were measured by $1 \mathrm{H}-\mathrm{NMR}$ and the samples were quenched with increasing linear-to-bottlebrush mass ratio. The products were dried with air-flow to remove unreacted monomers, dissolved in DCM to pass through neutral aluminum oxide columns, and vacuumed overnight. The degree of polymerization and mass ratio of linear end bocks were measured by 1H-NMR ( $\mathrm{CDCl} 3$, Brüker $400 \mathrm{MHz}$ spectrometer) as shown in Fig. S2 for PMMA-bbPDMS-PMMA. 


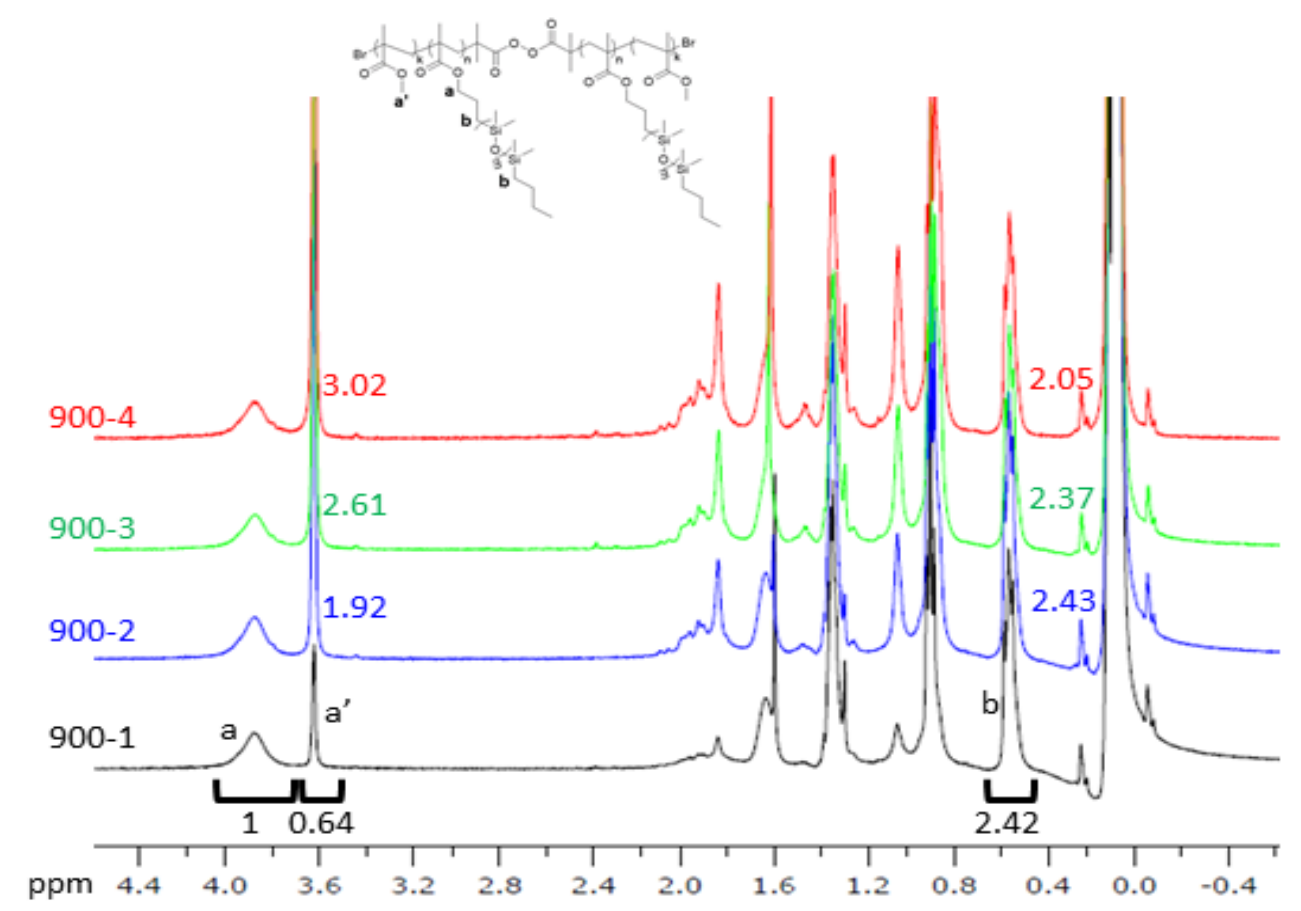

Fig. S2. ${ }^{1} \mathrm{H}-\mathrm{NMR}$ of PMMA-bbPDMS-PMMA, $\phi A=0.3,8,11$ and 14 (400 MHz, CDCl3): 3.9 (-CH2-O$\mathrm{C}=\mathrm{O}, \quad$ br, $\quad 2 \mathrm{H}), \quad 3.62 \quad$ (COO-CH3, s, $3 \mathrm{H}), \quad 0.54 \quad(\mathrm{COO}-\mathrm{CH} 2-\mathrm{CH} 2-\mathrm{CH} 2-\mathrm{Si}(\mathrm{CH} 3) 2, \quad \mathrm{t}, \quad 4 \mathrm{H})$. $D P M M A=\operatorname{Ar}\left(a^{\prime} / 3\right) / \operatorname{Area}(b / 4) * D P P D M S$.

\section{S2.1 Characterization.}

Atomic Force Microscopy. Monolayer samples were prepared with spin coating on the mica substrate. Thick films were prepared from concentrated solution in toluene followed by slow evaporation of the solvent. The imaging was performed in PeakForce QNM mode using a multimode AFM (Brüker) with a NanoScope V controller and silicon probes (resonance frequency of $50-90 \mathrm{~Hz}$ and spring constant of $\sim 0.4 \mathrm{~N} / \mathrm{m}$ ).

Mechanical tests. Dried samples were dissolved in toluene and poured into Teflon petri-dishes (Welch Fluorocarbon) and left to dry for 3 days. To prepare samples for mechanical measurements, the samples were gently removed from the dishes and punched into dumbbell samples with bridge dimensions of $12 \mathrm{~mm} \times 2 \mathrm{~mm} \times 1-2 \mathrm{~mm}$. Mechanical properties of samples were measured by RSA-G2 DMA (TA Instruments) in a constant Hencky strain rate of $0.003 \mathrm{~s}^{-1}$ until rupture occurred. Each sample was subjected to at least three tests to ensure accuracy of the measurements. The Origin 2016 software was used to analyze resulting stress strain curves by eq 2 . 

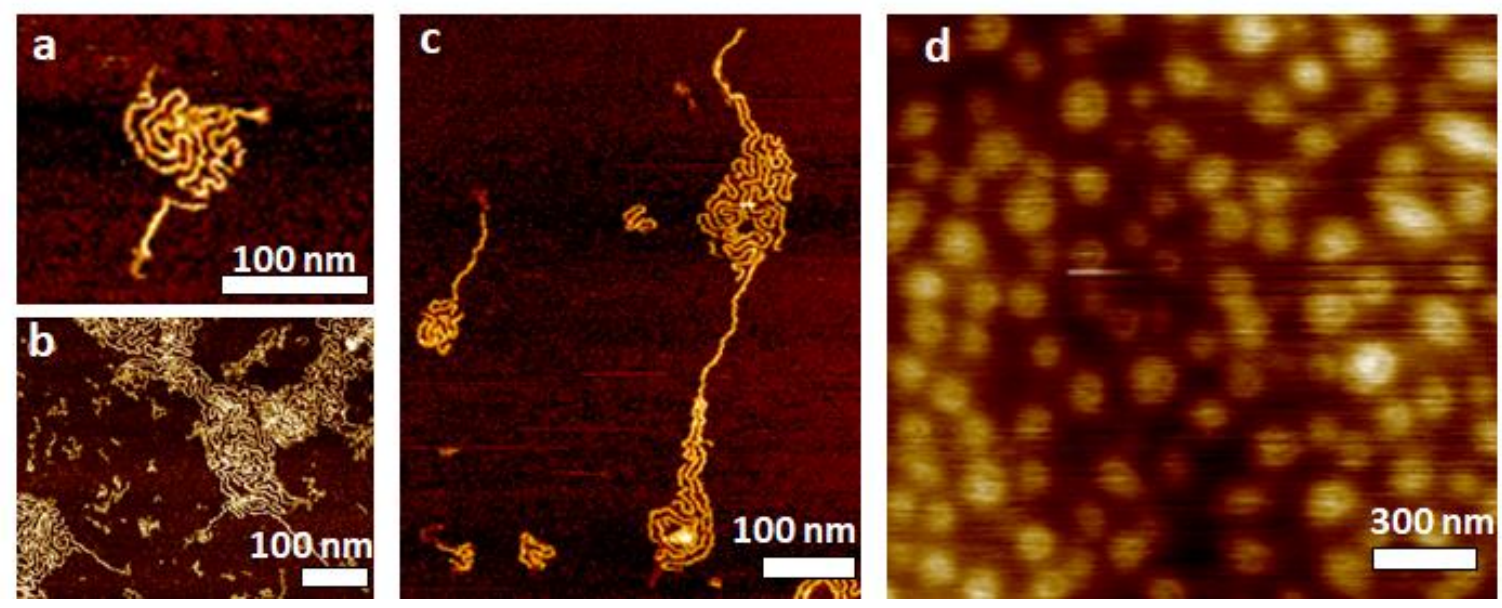

Fig. S4. AFM height micrographs of PMMA-bbPDMS-PMMA triblock copolymer (sample 900-4). (a-c) Individual molecules deposited by spincasting from dilute solutions on a mica substrate. The arrows point to linear blocks. (d) Surface morphology of thick films suggest microphase separation between PDMS bottlebrush blocks and PMMA linear blocks (spherical domains embedded in a PDMS matrix).

Electroactuation. A prestrain-free diaphragm was used to perform the electroactuation performance studied (Fig. S5). The casted plastomer films were placed between two flat facing acrylic rings with a 10 and $20 \mathrm{~mm}$ circular opening for the top and bottom ring, respectively. Two concentric circular carbon grease electrodes were painted onto opposite sides of the film and connected to an outside voltage source via copper tape. The sandwiched structure of acrylics ring/plastomers/acrylic ring screwed on top of a diaphragm chamber setup. To ensure out of plane deformation during plastomers actuation, a bias air pressure $P=5 \times 10^{-4}$ to $5 \times 10^{-3}$ atm was applied and controlled by a pressure regulator. The low bias pressure is low enough that does not induce ant prestrain to the plastomers. The active area of the DE films before actuation was circle with a diameter of $10.0 \mathrm{~mm}$.

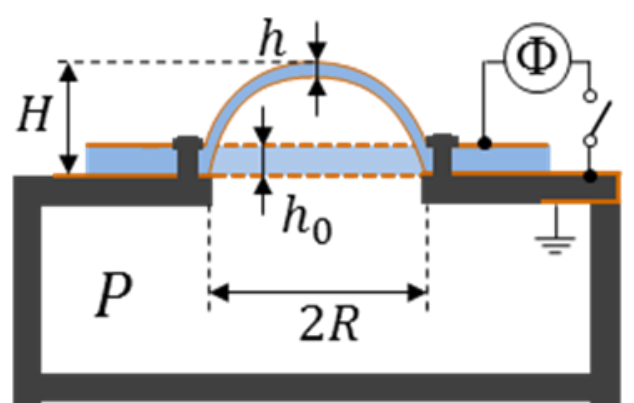

Figure S5: Schematics of electroactuation of a circular diaphragm. 
A high-voltage power supply (Gamma High Voltage Research Co.) was used to induce the actuation. Increases in voltage during actuation were measured with a voltmeter. Images and videos of actuations were recorded using a camera (Canon T4i, 18-55 mm lens). The actuation strain was calculated from the deformed film area as determined using side-profile images of the domes. Dimensions of the dome were calculated with ImageJ software. The nominal electric field was calculated by dividing the applied voltage by the initial thickness of the plastomer film.

Data analysis. From the uniaxial extension measurements of the plastomers the $\mathrm{G}$ and $\beta$ values for each sample were used to calculate the corresponding stress that the samples would generate under uniaxial compression $\left(\lambda=h / h_{0}<1\right)$ using the relationship established by Dobrynin et al. ${ }^{2}$

$$
\sigma_{\text {true }}(\lambda)=\frac{G}{3}\left(\lambda^{2}-\lambda^{-1}\right)\left(1+2\left(1-\frac{\beta\left(\lambda^{2}+\frac{2}{\lambda}\right)}{3}\right)^{-2}\right)
$$

The stress under uniaxial compression was then used to calculate the applied voltage, $\Phi$, required to achieved the same deformation observed in our diaphragm actuation setup using the preestablished relationship between the electromechanical pressure generated between the two electrodes and resisting mechanical force from the sample. ${ }^{3}$

$$
\Phi=\frac{h_{0}}{\sqrt{\varepsilon \varepsilon_{0}}} \sqrt{-\lambda^{2} \sigma_{\text {true }}(\lambda)-\frac{P R}{4 h_{0}} \frac{1}{\sqrt{\lambda^{-1}-1}}}
$$

$h_{0}$ corresponds to the thickness of the plastomer sample. $P$ and $R$ are the bias pressure used to buckle the sample upwards and the gas constant. The relative permittivity of the samples was taken to be the average permittivity of the sample based on the volume fraction of PDMS and MMA in each sample. The initial thickness $h_{0}$ is an average thickness of free-standing polymer film based on weight and area measurements. Thickness $h$ was calculated from eq 3 , where $H$ is obtained by image analysis considering a perfect hemisphere (Fig. S5).

$$
\frac{h_{0}}{h}=\frac{H^{2}+R^{2}}{R^{2}}
$$

Dielectric strength. The capacitor discharges when the capacitor field exceeds a critical electric field $E^{*}$, which gives the breakdown voltage as

$$
\Phi^{*}(\lambda)=\lambda h_{0} E^{*}
$$


Note that $E^{*}$ depends on experimental parameters including film thickness, inherent heterogeneities, and orientation of polymer chains. For example, the experimental data and theoretical predictions indicate that the dielectric breakdown (BD) increases with increasing crosslink density $\left(\sim n_{x}^{-1}\right)$, and Fig. $\mathbf{S 6}$ reveals that the BD likewise increases with decreasing film thickness.

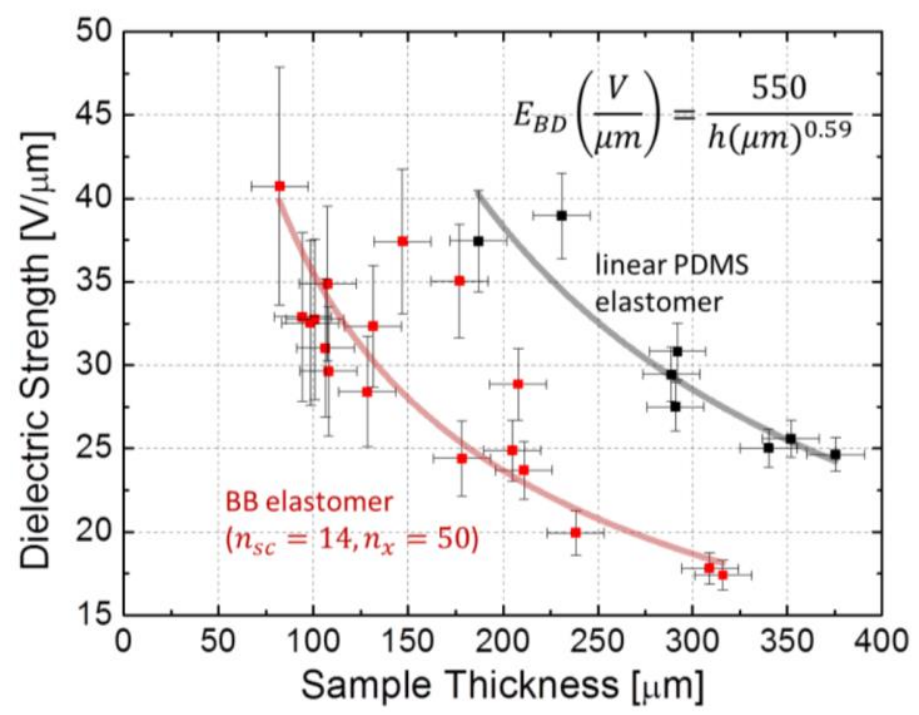

Fig. S6. Dielectric strength. The breakdown field of a PDMS bottlebrush elastomer was measured for as-cast films of different thicknesses $(h)$. A linear PDMS elastomer was also measured as a reference sample. The fitting equation in the upper-right corner corresponds to the bottlebrush sample. For a typical $h_{0}=0.44 \mathrm{~mm}$ and $\lambda_{a}^{B D} \cong 4.0$, the breakdown occurs at an electric field of 33

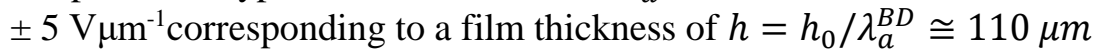

1. Vatankhah-Varnosfaderani, M.; Keith, A. N.; Cong, Y.; Liang, H.; Rosenthal, M.; Sztucki, M.; Clair, C.; Magonov, S.; Ivanov, D. A.; Dobrynin, A. V. Chameleon-like elastomers with molecularly encoded strain-adaptive stiffening and coloration. Science 2018, 359, (6383), 1509-1513.

2. Dobrynin, A. V.; Carrillo, J.-M. Y. Universality in nonlinear elasticity of biological and polymeric networks and gels. Macromolecules 2010, 44, (1), 140-146.

3. Vatankhah-Varnoosfaderani, M.; Daniel, W. F.; Zhushma, A. P.; Li, Q.; Morgan, B. J.; Matyjaszewski, K.; Armstrong, D. P.; Spontak, R. J.; Dobrynin, A. V.; Sheiko, S. S. Bottlebrush elastomers: A new platform for freestanding electroactuation. Advanced Materials 2017, 29, (2), 1604209. 